\title{
Experimental Study of Mechanical Hysteresis of NiTi During Ferroelastic and Superelastic Deformation
}

\author{
L. Orgéas, Y. Liu* and D. Favier \\ Laboratoire Sols Solides Structures (3S), UMR 5521 du CNRS, Université Joseph Fourier, BP. 53X, \\ 38041 Grenoble cedex, France \\ * Department of Mechanical and Materials Engineering, University of Western Australia, Nedlands, \\ WA 6907, Australia
}

\begin{abstract}
Shape memory alloys are known to exhibit a range of novel thermomechanical behaviour associated with the unique thermoelastic martensitic transformation. Such behaviour includes the superelasticity associated with stress-induced martensitic transformation at relatively high temperatures and the ferroelasticity associated with a martensite reorientation process at low temperatures. Both the stress-induced martensitic transformation and the martensite reorientation are energy-dissipative processes, i.e., hysteretic between the forward and reverse processes. This work was aimed at studying the hysteretic behaviour of the ferroelasticity and superelasticity observed in a polycrystalline NiTi alloy by carrying out simple shear deformation tests through both major and subloop cycles. It was found that subloops are always closed and enclosed inside the major loop and that the stress hysteresis of a subloop is only dependent on the strain amplitude of the subloop, regardless of the position of the subloop inside the major loop.
\end{abstract}

\section{INTRODUCTION}

Shape memory alloys (SMAs) are known to exhibit a range of novel thermomechanical properties due to thermoelastic martensitic transformations, a particular type of first order, displacive, and largely deviatoric solid-solid phase transformation. When deformed at certain temperatures above $A_{f}$, the finishing temperature of the reverse martensite-to-austenite transformation, SMAs may display superelastic behaviour, which is a quasi-elastic deformation far beyond the conventional elastic limit of the material. If the temperature is lowered, superelasticity vanishes and the deformation proceeds by another interesting mode known as the ferroelasticity, in recognition of its phenomenological similarity to ferromagnetism. The superelasticity exhibited by SMAs is associated with stress-induced martensitic transformations and the ferroelasticity, on the other hand, is associated with a martensite reorientation process. As many natural phenomena, martensitic transformation and martensite reorientation are not perfectly reversible physical processes, so that there always occurs a hysteresis between the forward and reverse processes during thermal or mechanical cycling. Considering quasi-static evolutions, microstructural reasons of such an irreversible behaviour are often attributed to the moving interfaces of martensite variants being slowed down by internal defaults such as grain boundaries, dislocations and precipitates [1]. Among the numerous experimental work performed in the past three decades that aimed at characterising the thermo- mechanical behaviour of SMAs, some have been devoted to study the complex hysteretic behaviour of these materials. In this respect, the majority of the studies have focused their attention on Cu-based SMAs, e.g., characterisation of hysteretic behaviour of $\mathrm{Cu}$-based SMAs during thermal cycling [2-5], superelastic cycling [69] and ferroelastic cycling [10] using both single-crystal and polycrystalline specimens. To the contrast, much fewer experimental studies have been devoted to NiTi alloys [11-12]. The aim of all these experimental works has been to draw general rules governing the hysteretic behaviour of these alloys [7,11]. The understanding of these rules is fundamentally important for the study of SMAs in two respects. First, it improves the understanding of the mechanisms of the physical phenomena of shape memory effect. Secondly, it provides experimental evidences and theoretical framework for the establishment of constitutive models which take into account the effect of hysteresis on the thermomechanical response of SMAs. This is in turn crucially important for the successful application of SMAs. Frequently SMAs are utilised in practical applications in incomplete transformation or reorientation cycles. Therefore, more experimental work and theoretical analyses are required to accomplish a complete and systematic understanding of the hysteretic behaviour of thermo-elastic martensitic transformations. This work is aimed at studying the 
mechanical hysteretic behaviour of an equiatomic NiTi SMA and achieving an understanding of the rules governing the hysteresis of this alloy by carrying out mechanical testing in both ferro- and superelasticity.

\section{EXPERIMENTAL PROCEDURE}

The mechanical hysteresis of NiTi was to be studied in both superelastic and ferroelastic modes. Conventionally, most mechanical testing has been carried out in tension using wire specimens. For nearequiatomic NiTi alloy wire samples tested in tension, however, Lüders-type deformation behaviour has often been observed during stress-induced martensitic transformation and martensite reorientation processes [14]. The occurrence of Lüders-type deformation is detrimental to the study of mechanical hysteresis, because in this case the strain state inside the gauge section of a sample is inhomogeneous [15]. This problem can be avoided in shear test using plate samples, in which it has been observed that the deformation proceeds in a macroscopically uniform manner [13]. Shear testing has another advantage over tensile testing of wire samples. It allows easy reversion of the direction of loading and the hysteretic behaviour in both directions is symmetric, in contrast to the asymmetric stress-strain curves observed in tension-compression tests [16]. Furthermore, symmetric cycling in both the forward and reverse loading directions is an essential condition for the measurement of stress hysteresis for ferroelasticity. Therefore, simple shear was chosen to be the deformation mode for both ferroelastic and superelastic testing.

The material used was a $\mathrm{Ni}_{50} \mathrm{Ti}_{50}$ alloy supplied in a sheet form by Memometal Industry (France). The asreceived sheets were first solution treated at $1213 \mathrm{~K}$ for $1.8 \mathrm{ks}$, followed by quenching into water at room temperature. Two solution-treated sheets were cold-rolled to over $20 \%$ in thickness reduction and then annealed for $1.8 \mathrm{ks}$ at $603 \mathrm{~K}$ and $705 \mathrm{~K}$, respectively. Both sheets exhibited optimum superelasticity and excellent ferroelasticity. Shear samples for ferroelastic and superelastic testing were cut from the heattreated sheets with a geometry of $30 \times 20 \times 1 \mathrm{~mm}^{3}$. The gauge section of the shear samples was $30 \times 3 \times 1 \mathrm{~mm}^{3}$ in dimension with the shear direction being along the length of the samples. The oxide surface on the samples resulting from previous heat treatment was removed mechanically using SiC abrasive paper prior to testing.

Superelastic and ferroelastic testings were performed in simple shear using a shear device on an AdamelMTS DY35 universal mechanical testing machine [13]. The shear device is equipped with a liquid bath which enables the temperature of the sample to be controlled during testing with an accuracy of $0.1 \mathrm{~K}$. This device also allows local measurement of the shear strain using a displacement extensometer placed near the sample. A low shear strain rate of $[\mathrm{d} \gamma / \mathrm{dt}]=1.7 \times 10^{-3} \mathrm{~s}^{-1}$ was adopted. Under this strain rate the effect of the release and absorption of the latent heat associated with stress-induced martensitic transformation was minimised and the testing condition could be considered practically isothermal. In order to have a consistent measurement of mechanical hysteresis, specimens for both superelastic and ferroelastic testing were pre-cycled in their respective mode for a number of symmetric cycles to stabilise the stressstrain behaviour prior to measurement. It is known that the stress-strain behaviour of SMAs is subjected to modifications during mechanical cycling [17], including the changes in hysteresis, changes of the shape of the stress-strain curves, deterioration of superelastic recovery and the accumulation of permanent deformation. These modifications are observed to occur at a decreasing rate and to saturate after a number of cycles. Hence pre-cycling was performed symmetrically about the zero-strain position to a strain amplitude well within the shear transformation strain limit of the material. After the stabilisation cycling, hysteresis was measured for subloops of different strain amplitudes ranging from $\Delta \gamma=0.75 \%$ to $12 \%$. Between each two subloop cycles a full loop was performed to ensure an identical starting condition for the subloops.

\section{RESULTS}

\subsection{Symmetric cycling}

The adoption of simple shear as the deformation mode allows the mechanical cycling to be performed symmetrically about the zero-strain point in both forward and reverse loading directions. Symmetric cycling is important for both the stabilisation of the stress-strain behaviour and the measurement of 
mechanical hysteresis: Figure 1 shows a selection of superelastic cycles of a NiTi sample, which was cycled to $+7 \%$ under unidirectional loading for 50 cycles and then cycled for another 70 cycles to $\pm 7 \%$ under symmetric loading condition. In this figure curve (a) is the initial unidirectional cycle, curve (b) is the 50th cycle of the unidirectional cycling, curve (c) is the symmetric cycle immediately following curve (b), and curve (d) is the 70th symmetric cycle. It was observed that a "complete" superelastic recovery, i.e., a closed superelastic loop, was obtained after 50 unidirectional cycles at the expense of the accumulation of a remanent strain. The remanent strain is attributed to two contributions: true plastic deformation and stabilised martensite. Expanding the superelastic cycling at this stage to the negative deformation direction by reversing the shear load resulted in a symmetric superelastic loop. It is evident that the stress hysteresis of the unidirectional, closed superelastic loop (curve (b)) is much smaller than the stress hysteresis of the symmetric superelastic loop at the same strain position (curve (c)). Moreover, comparison between curves (b) and (d) indicates that the symmetric cycling resulted in a partial recovery of the remanent strain developed during the previous unidirectional cycling. This is attributed to the rejoin of the stabilised martensite in the superelastic transformation process. These observations demonstrate that such centred symmetric mechanical cycling is important and beneficial in achieving a stabilised stress-strain behaviour without a significant loss of superelastic recovery.

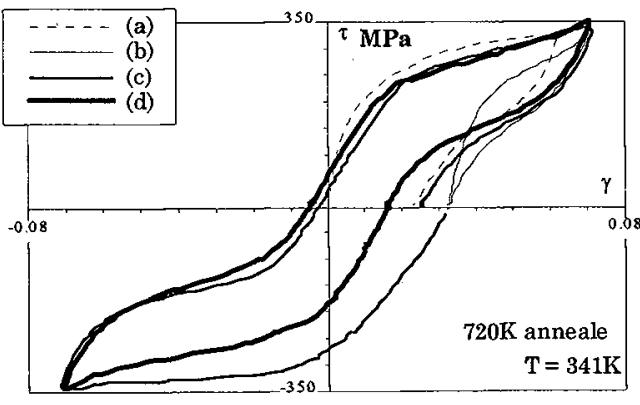

Figure $1:$ Influence of the loading path on the hysteresis study

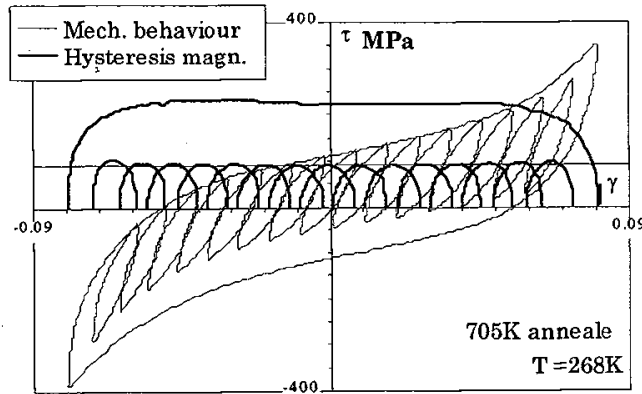

Figure 2 : Subloops and hysteresis magnitude with $\Delta \gamma=2 \%$ in ferroelasticity

\subsection{Hysteresis in ferroelasticity}

To stabilise the ferroelastic behaviour, a sample was cycled between $\pm 8 \%$ of strain at $268 \mathrm{~K}, 8 \mathrm{~K}$ below the $\mathrm{A}_{\mathrm{s}}$ temperature, for 100 cycles. The sample was cooled in liquid nitrogen prior to cycling to ensure a complete martensitic structure. After the stabilisation cycling, subloops with $\Delta \gamma=2 \%$ were performed at different values of the initial strain $\gamma^{\mathrm{R}}$, as shown in Figure 2. In this figure the full loop cycles performed after each subloop cycle were omitted for simplicity, except the first one. It is seen that all the subloops were entirely enclosed inside the major loop. The stress hystereses of the subloops as well as that of the major loop were measured after removing the effect of elastic deformation using a modulus of elasticity in shear of $30 \mathrm{GPa}$. The results of the measurement are shown in Figure 2 too. It is seen that the stress hysteresis, $\Delta \tau_{\text {hys }}$, of the major loop increased rapidly at each end of the loop and approached a saturation value towards the centre. The stress hysteresis of a subloop reached a maximum in the middle of its strain span. The maximum stress hysteresis of the subloops, $\Delta \tau_{\text {hysmax }}$, was found to be independent of the position where a subloop was performed and to be constant at $\approx 90 \mathrm{MPa}$. Measurements of subloops of different strain amplitudes are shown in Figures 3 and 4, where the start-point of the subloops $\gamma^{R}$ were at $\gamma_{\max }$ and 0 , respectively. The results of the measurement of stress hysteresis indicated that $\Delta \tau_{\text {hysmax }}$ was a function of the strain magnitude, $\Delta \gamma$, of a subloop and that increasing $\Delta \gamma$ led to an increase in $\Delta \tau_{\text {hysmax }}$ independently of $\gamma^{R}$.

\subsection{Hysteresis in superelasticity}

The same experimental procedure as for the measurement of ferroelasticity was carried out for the measurement of superelasticity, except the testing temperature being $333 \mathrm{~K}, 4 \mathrm{~K}$ above the Af temperature of the material. Measurements of subloops with $\Delta \gamma=1.5 \%$ and $\Delta \gamma=2 \%$ are shown in Figures 5 and 6 , 
respectively. It was found that the subloops were always closed and that $\Delta \tau_{\text {hysmax }}$ of the subloops was independent of the position on the major loop and constant for subloops of the same $\Delta \gamma . \Delta \tau_{\text {hysmax }}$ was measured to be $55 \mathrm{MPa}$ for subloops of $\Delta \gamma=1.5 \%$ and $67 \mathrm{MPa}$ for $\Delta \gamma=2 \%$. Measurements of subloops of different strain amplitudes $\Delta \gamma$ are shown in Figures 7 and 8. The dependence of hysteresis on $\Delta \gamma$ for superelasticity was found to be similar to that for ferroelasticity, as shown in Figures 3 and 4.

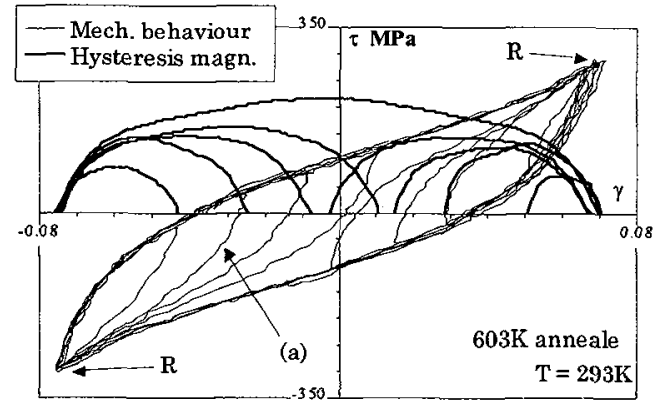

Figure 3 : Subloops and hysteresis magnitude with increasing values of $\Delta \gamma$ in ferroelasticity, start point at $\gamma=\gamma_{\max }$.

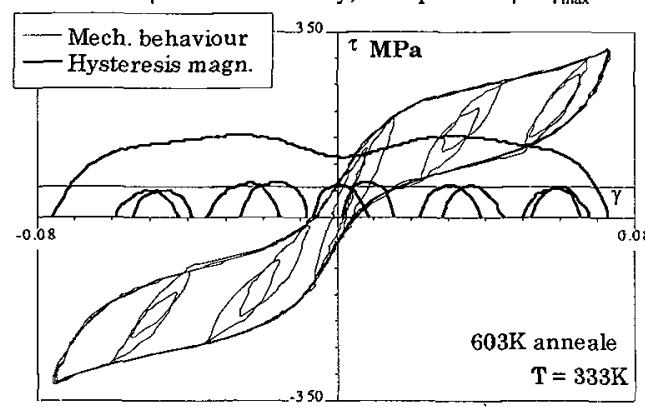

Figure 5 : Subloops and hysteresis magnitude with $\Delta \gamma=1.5 \%$ in superelasticity.

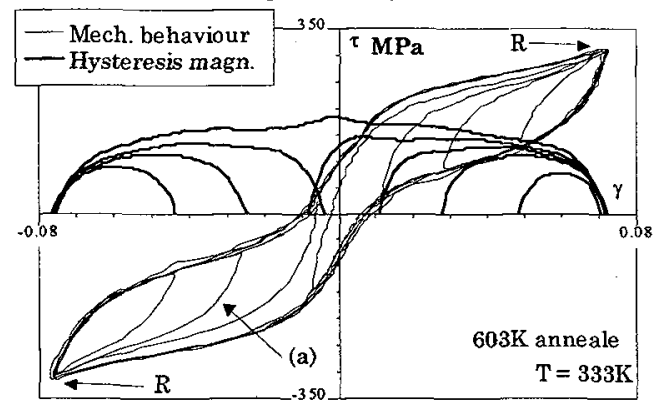

Figure 7 : Subloops and hysteresis magnitude with increasing values of $\Delta \gamma$ in superelasticity, start point at $\gamma=\gamma_{\max }$.

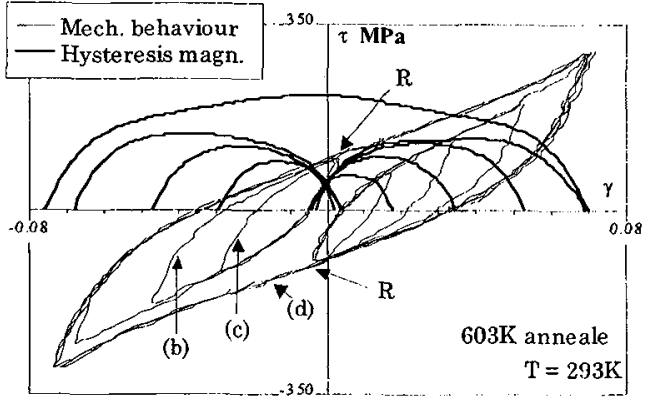

Figure 4 : Subloops and hysteresis magnitude with increasing values of $\Delta \gamma$ in ferrroelasticity, start point at $\gamma=0$.

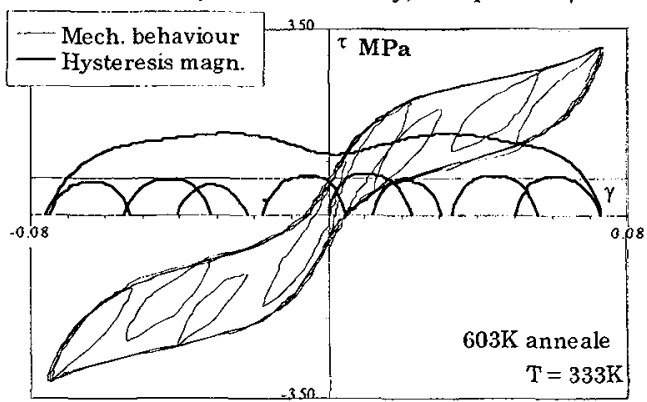

Figure 6 : Subloops and hysteresis magnitude with $\Delta \gamma=2 \%$ in superelasticity.

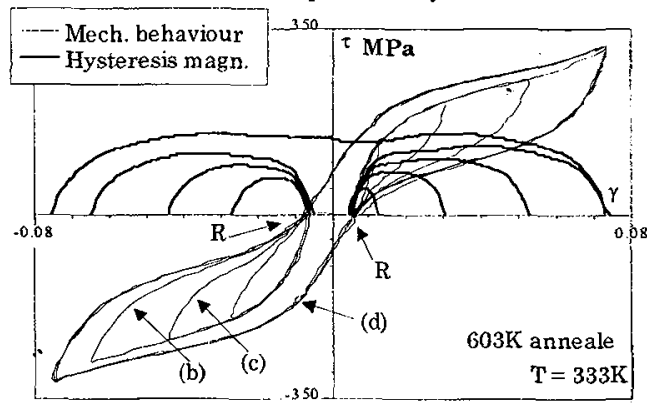

Figure 8 : Subloops and hysteresis magnitude with increasing values of $\Delta \gamma$ in superelasticity, start point at $\tau=0$.

\subsection{Closure of subloops}

To verify the concept of "erasable micromemory"[8] and the notation of "discrete memory" introduced by Guélin [18], a specially designed partial cycling test was carried out on ferroelasticity and superelasticity, as shown in Figures 9 and 10, respectively. In this experiment the major loop cycling was performed symmetrically with a strain amplitude of $\Delta \gamma= \pm 11 \%$ for both the ferroelasticity and superelasticity. The partial loop cycling started at point 1 and continued following the numerical order of the return points marked in the figures. It was observed that the stress-strain curve in section 6-7 passed through all the previous return point, e.g., points 5,3 and 1 , in both cases of ferroelasticity and superelasticity, closing all the incomplete partial loops. This observation confirms the concept of "erasable micromemory", which is also 
known as the "return point memory" effect [6]. The re-assembling of the return points along the path seems to suggest that the thermomechanical behaviour of a shape memory alloy is dictated by its previous deformation history.

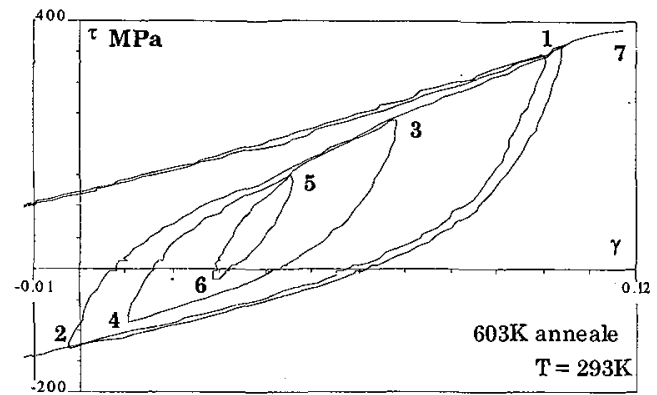

Figure 9 : Discrete memory concept in ferroelasticity

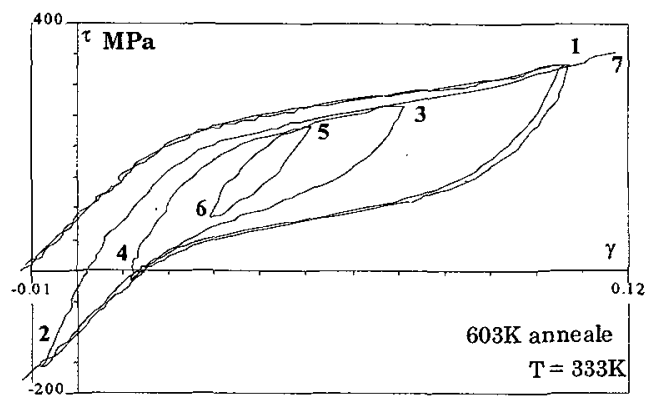

Figure 10 : Discrete memory concept in superelasticity

\section{DISCUSSION}

The experimental results reported above reveal a close parallelism between the irreversible nature of the two mechanical deformation modes in SMAs [19]. The irreversible nature of these two processes appears to respect a common set of phenomenological rules [11], which are reformulated and summarised as following. For the reason of simplicity, "loop" is used in the following discussion to indicate both ferroelastic loop and superelastic loop, because these rules apply to both situations.

\subsection{Mechanical hysteresis}

(1) Both ferroelasticity and superelasticity exhibit mechanical hystereses, even at very small strain amplitudes, e.g., a maximum stress hysteresis of $\Delta \tau_{\text {hysmax }}=45 \mathrm{MPa}$ was measured for a superelastic loop of $\Delta \gamma=0.75 \%$. The occurrence of such hysteresis appears to be irrespective to either the strain amplitude of the stress-strain loop or at which end the loop is started. In fact, small mechanical hystereses have been measured between simple unloading and reloading curves for shape memory alloys [20]. The apparent inelasticity has been attributed to simultaneous detwinning of martensite variants from the fully oriented configuration in ferroelasticity $r$ possibly a simultaneous reverse transformation of stress-induced martensite in superelasticity.

(2) The magnitude of the hysteresis of a loop is independent of the pre-deformation, $\gamma^{\mathrm{R}}$, from which the loop is performed, as evident in Figure 2 for ferroelasticity and Figure 6 for superelasticity. This statement is valid when the pre-deformation is within the limit of the transformation strain associated with the formation of martensite. The magnitude of hysteresis is only dependent on the strain amplitude of the loop, $\Delta \gamma$, and increases with increasing $\Delta \gamma$ (Figures 11 and 12). The maximum magnitude of stress hysteresis appears to be qualitatively consistent for the two deformation modes, being $\approx 250 \mathrm{MPa}$ for ferroelasticity and $\approx 220 \mathrm{MPa}$ for superelasticity respectively. That the mechanical hysteresis is qualitatively the same for stress-induced martensitic transformation and for martensite reorientation is an important observation. It suggests that microstructural events which cause mechanical irreversibility to ferroelasticity and superelasticity are mainly independent of the deformation mode.

\subsection{Return-point memory and erasable micromemory}

(3) A loop always exhibits return-point memory. This phenomenon is illustrated in Figures 3 and 4 for ferroelasticity and Figures 7 and 8 for superelasticity. For a subloop started from the extremity of the major loop, curve (a) in Figure 3 for example, whereas the upper branch of the subloop overlaps with the major loop, the lower branch returns to the major loop only at the return point $R$, regardless of the strain amplitude of the subloop (Figures 3 and 7). This return-point memory also prevails when a subloop is started by returning from the middle of the major loop, as shown in Figures 4 and 8 . This return-point has been regarded as the common reference state $\left(\tau^{R}, \gamma^{R}\right)$ [18] for the subloops, marked in Figures $3,4,7$ and 8 .

(4) This effect is further demonstrated in Figures 9 and 10. In these figures point 6 is approached by retur- 
ning from points $1,2,3,4$ and 5 sequentially. In this regard loop $\{1-2-1\}$ may be viewed as the parent loop of loop $\{3-4-3\}$, which in turn is the parent loop of loop $\{5-6-5\}$. Reversing loading at point 6 returns to point 5 , as expected. However, continued loading extends the stress-strain curve to point 3 and point 1 sequentially.These two points are memorised only because they had served as the return points in the stressstrain history when point 6 was approached. This observation immediately suggests two conclu-sions: (1) not only the return point of the subloop but also the return points of previous parent loops are memorised, and (2) once a return point is memorised for a subloop the stress-strain curve resumes the pa-rent loop, as if the closed subloop had been forgotten. The later has in fact been recognised as the "erasable micromemory effect" to indicate the ineffectiveness of a subloop to the return-point memory of its parent loop. (5) A consequence of this erasable micromemory effect is the change of the reference state, for example from $\left(\tau^{6}, \gamma^{6}\right)$ to $\left(\tau^{4}, \gamma^{4}\right)$ and then to $\left(\tau^{2}, \gamma^{2}\right)$ when the stress-strain curve travels along the path from point 6 to points 5,3 and 1 sequentially. This discrete change of reference state is also known as the "discrete memory effect" [18].

+

\subsection{Hierarchisation of loops and subloops}

(6) When a small loop is performed within the strain span of a large loop, it is always contained completely inside the large loop. This is in fact phenomenologically determined by rule 2 and rule 3 stated above, as explained below. Taking subloop (a) in Figure 3 (or 7) for example, the "return-point memory effect" determines that the upper branch of the subloop overlaps the upper branch of the enveloping parent loop and the strain amplitude dependence of the magnitude of hysteresis determines that the lower branch of the subloop is confined within the lower branch of the parent loop. Applying the same rules, subloop (c) performed from subloop (b) in Figure 4 (or 8 ) is thus completely contained inside the major loop (d).

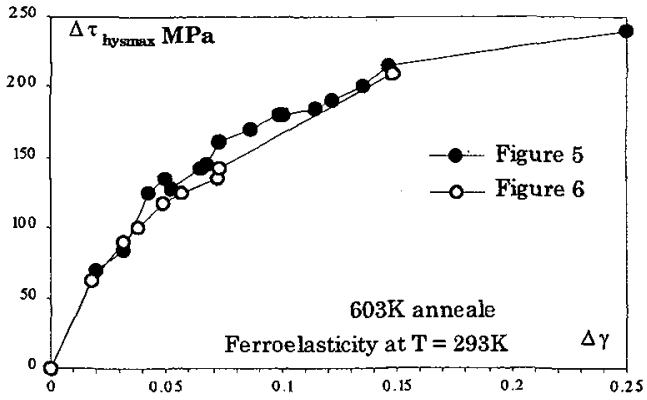

Figure $11: \Delta \tau_{\text {hysmax }}(\Delta \gamma)$ in ferroelasticity

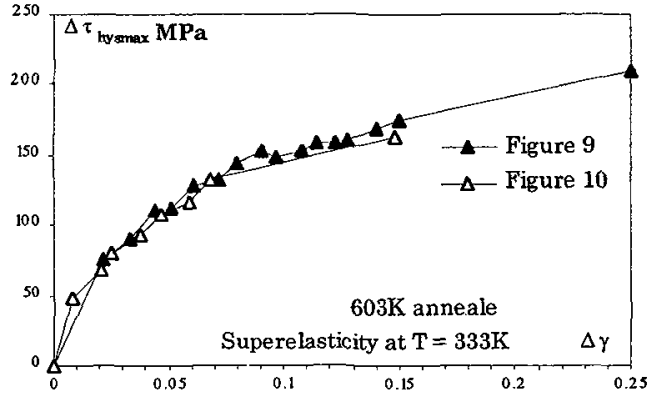

Figure $12: \Delta \tau_{\text {hysmax }}(\Delta y)$ in superelasticity

\section{References}

[1] Delaey L. and Aernoudt E., Proc. ICOMAT 86, JIM, (1986), 926-933.

[2] Planes A., Castan T., Ortin J. and Delaey L., J. Appl. Phys., 66, (1989), 2342-2348.

[3] Cornelis I. and Wayman C.M., Scripta Met., 10, (1976), 359-364.

[4] Amengual A., Cesari E. and Segui C., Proc of ICOMAT 92, Monterey, CA, USA (1992), 377-382.

[5] Lü L., Aernoudt E. and Delaey L., Scripta Met., 22, (1988), 1435-1440.

[6] Ortin J., J. Appl. Phys, 71, 3, (1992), 1454-1461.

[7] Fu S., Huo Y. and Müller I., Acta Mech., 99, (1993), 1-19.

[8] Vergut H., Delaey L. Aernoudt E. and Vermeesch W. Proc. Euromech Col., Warzava, Poland, (1987).

[9] Tourabi A., Wack B. and Favier D., Mat. Sci. Forum, 56-58, (1990), 535-540.

[10] Müller I. and Zak G., J. de Phys. III, C8, 5, (1995), 197-201.

[11] Cory J.S. and McNichols Jr J.L., J. Appl. Phys., 58, n 9, (1985), 3282-3294.

[12] Manach P.Y., Ph.D. Thesis, INPG, France, (1993).

[13] Manach P.Y. and Favier D., in press in Mat. Sci. and Eng., (1997).

[14] Liu Y., Orgéas L. and Favier D., J. de Phys. III, C8, 5, (1995), 593-598.

[15] Shaw J.A. and Kyriakides S., J. of Mech. Phys. Solids, 43, 8, (1995), 1243-1288.

[16] Orgéas L. and Favier D., J. de Phys. III, C8, 5, (1995), 605-610.

[17] Miyazaki S., Imai T., Jgo Y. and Otsuka K., Met. Trans. A, 17, (1986), 115.

[18] Guélin P., J. de Mécanique, 19, 2, (1980), 217-247.

[19] Delaey L., Krishnan R.V., Tas H. and Warlimont H., J. Mat. Sci., vol 9, (1974), 1521-1535.

[20] Duerig T.W. and Zadno R:, Engineering Aspects of SMAs, Butterworth-Heinemann, London (1990), 349. 